TITRE: LE VAGUE COMME TERRAIN D'OBSERVATION DE DISCOURS HORS-NORMES

Auteur(s): PAscale Brunner, MaîTRe de conférences, Université de PoItIERS, FORELL

PUBLICATION: ÉCRITS HORS-NORMES

PAGES: $79-91$

DiRecteurs: Agnès Steuckardt et Karine Collette

ÉdITEUR: LES ÉDITIONS DE L'UNIVERSITÉ DE SHERBROOKE, 2019

ISBN: 978-2-7622-0360-8

URI: HTTP://HDL.HANDLE.NET/11143/15574

DOI: HTTPS://DOI.ORG/10.17118/11143/15574 


\section{Le vague comme terrain d'observation de discours hors-normes}

Pascale Brunner, Maître de conférences

Université de Poitiers, FORELL

Résumé : Cette étude est consacrée aux représentations qui circulent dans le discours quotidien à propos du vague. Ce dernier étant considéré comme allant à l'encontre du bon usage clair et précis de la langue - et se situant donc hors-normes - nous allons dans un premier temps confronter représentations scientifiques et populaires à propos de ce phénomène. À l'aide d'un corpus d'articles de presse rassemblés autour de la séquence évaluative "c'est vague », on avancera dans un deuxième temps quelques hypothèses qui tentent d'expliquer cette virulente critique que rencontre le vague dans la langue française.

Mots clefs : vague, précision, représentation, locuteur ordinaire, évaluation

Abstract : This study inquires the representations one can find in ordinary discourse regarding vague language. As the phenomenon is often criticized because it goes against good, clear and precise usage of language, thus considered as being "hors-normes" (non-standard), we first will confront scientific versus popular beliefs about vague language. Based on a corpus of French press articles which all contain the evaluative sequence "it's vague", we will then propose some hypotheses that might explain the strong criticism which vagueness encounters in the French language.

Key words : vagueness, precision, representation, ordinary speaker, evaluation 
Cet article se consacre à l'étude de la représentation qu'ont la plupart des gens d'un parler qui, de par sa précision et sa clarté, correspondrait à un imaginaire de langue idéale (Wilmet, 1987). Les ouvrages qui traitent du vague dans la langue le mentionnent parfois explicitement:

People have many beliefs about language. One important one is that "good" usage involves (among other things) clarity and precision. Hence, it is believed that vagueness, ambiguity, imprecision, and general woolliness are to be avoided. (Channell, $1994: 1$ ).

Les croyances populaires - les « beliefs » - sembleraient, concernant le « bon usage » de la langue, impliquer clarté et précision. Tout usage approximatif de la langue allant à l'encontre de cet idéal de clarté, de précision («Ce qui n’est pas clair n’est pas français », selon Rivarol) se situe, par conséquent, hors-normes.

Mais de quelle «norme», et par conséquence de quel « hors-normes » parle-t-on ici ? Qui érige cette norme du précis : les locuteurs d'une communauté linguistique? Les institutions chargées de la « sauvegarde » et de la «normalisation » de la langue française ? Et qu'en disent les « savants », les linguistes?

Nous confronterons dans un premier temps le positionnement du chercheur d'une part, et celui du locuteur ordinaire ${ }^{1}$ d'autre part vis-à-vis du vague. La deuxième catégorie de locuteurs jugeant le vague avec davantage de critique que la première, nous tenterons, dans un deuxième temps, d'élucider les raisons qui amènent le locuteur ordinaire à censurer le vague de manière si rigoureuse. Nous mènerons une analyse sémantico-pragmatique de l'usage du mot vague en contexte dans un corpus médiatique pour comprendre les sens avec lesquels les locuteurs utilisent ce terme. En mettant au jour le dénominateur commun entre ces différents sens et usages, on pourra donner une première explication à cet acharnement contre le vague.

Dans un troisième mouvement, on essaiera de relier ces représentations à des « causalités institutionnelles, culturelles ou historiques » (von Münchow et Rakotonoelina, 2006). Le mythe de la clarté de la langue en tant que discours circulant pourrait-il par exemple encadrer, encore aujourd'hui, la perception du vague et les caractéristiques péjoratives qui y sont associées? Cela restera certes une hypothèse, mais elle mérite d'être évoquée lorsque l'on étudie le vague auprès d'une langue dont l'histoire a mobilisé à travers les siècles de multiples positionnements normatifs, voire puristes à son sujet.

\section{Deux catégories de locuteurs, deux types de jugement}

La norme pouvant étant considérée comme un ensemble de points de vue (qu'ils soient descriptifs ou prescriptifs) portés sur les différentes formes de la langue, il convient de s'interroger non seulement sur la manière dont les linguistes conçoivent un phénomène linguistique, mais également sur le point de vue du locuteur ordinaire qui est quotidiennement confronté à la langue et émet des commentaires métalinguistiques à son égard. Quels sont les éléments langagiers que les uns et les autres, linguistes et non linguistes, considèrent comme vagues et comment précisément ces derniers se positionnent-ils vis-à-vis d'eux?

1. C'est-à-dire le locuteur non linguiste ou le linguiste en tant que locuteur tel qu'il l'est en dehors de son activité de recherche. 
Notre étude s'inscrit ainsi dans la tendance actuelle de donner plus de poids aux savoirs profanes des locuteurs ordinaires² (Paveau, 2008 ; Lecolle, 2014 ; Brunner et al. à paraître). Elle s'intéresse à la perception, au sentiment linguistique ou à ce que Cécile Canut (2007 : 51) conçoit comme " activité épilinguistique » émergeant dans « tout type de discours autonome sur les langues ou les pratiques ${ }^{3}$ ». II s'agira alors d'interroger le rapport entre un imaginaire du hors-normes et une réalité linguistique d'une part, et de confronter ce rapport aux approches savantes d'autre part - étude qui pourra s'inscrire dans une réflexion plus large autour de la conception du hors-normes appréhendé par différents «types » de locuteurs (savants et non-savants).

\section{Le point de vue du chercheur : le vague,propriété inhérente à la langue et usage bénéfique}

Nous commençons par un bref tour d'horizon des différentes approches « savantes » vis-à-vis du phénomène qui nous intéresse ici : comment les philosophes, sémanticiens et chercheurs en pragmatique conçoivent-ils le vague et surtout quels sont les éléments langagiers considérés comme tels?

Le vague est un phénomène aux multiples facettes : il peut se situer dans le monde, dans la perception de celui-ci, dans les pensées que nous en avons, mais aussi à l'intérieur du langage et plus particulièrement dans l'usage que nous en faisons. Ces différentes possibilités témoignent de la complexité du phénomène et expliquent la diversification des disciplines qui se fixent pour objectif de cerner la notion du vague.

Les chercheurs qui s'inscrivent dans une approche logico-philosophique (Williamson, 1994 ; Keefe, 2000) appréhendent le vague en tant que phénomène qui présente des « cas-limites » dans l'extension d'un terme et qui défient ainsi la logique classique bivalente. Dans cette perspective, les catégories linguistiques typiquement vagues sont celles des prédicats graduables tels que chauve, rouge, tas, etc. puisque certaines configurations dans la réalité (une personne n'ayant plus que trois cheveux sur le crâne) rendent l'assignation du prédicat (chauve ou non chauve) délicate. La propriété d'une expression vague est ainsi définie comme « un concept dont l'extension n'est pas strictement délimitée, c'est-à-dire tel qu'il existe des objets à propos desquels la question de savoir s'ils satisfont le concept en question reste indécidable»(Blay, 2003 : 434).

Les linguistes qui s'inscrivent dans le domaine sémantique semblent à leur tour avoir du mal à s'accorder à la fois sur une définition du vague et sur ce qui le sépare de phénomènes apparentés. Dönninghaus (2005: 155 et suiv.) et Mihatsch (2010 : chap.1) présentent les multiples phénomènes d'indétermination recensés dans la langue : généricité, approximation, sous-détermination, ambiguité, etc. Les discussions menées concernent souvent la délimitation entre ces différents phénomènes à l'exemple de Kleiber (1987) et Fuchs (1986) qui se penchent sur les caractéristiques distinguant le vague de l'ambigu: dans les deux cas il y a indétermination quant à l'interprétation mais dans le cas de l'ambiguïté, l'indétermination concerne l'interprétation à choisir ; dans le cas du vague, il y a indétermination quant à l'applicabilité référentielle.

2. Nous faisons référence à la folk linguistique (Niedzielski et Preston, 2000), discipline qui s'intéresse en particulier aux productions métalinguistiques du sujet parlant non spécialiste et qui les considère comme étant légitimes, reconnaissables comme tels et pleinement intégrables en tant que données linguistiques (Paveau, 2008).

3. Cécile Canut reprend et élargit le sens initial de la notion d'« épilinguistique », attribuée à Culioli (1991), recouvrant chez lui l'activité non consciente qui génère des représentations mentales à propos du monde et du langage qui peuvent à leur tour influencer la production verbale. 
Les études à orientation pragmatique (Channell, 1994 ; Overstreet, 2005 ; Jucker et al., 2003) centrent leur intérêt davantage sur l'examen de l'usage des expressions considérées comme vagues : les hedges (genre de), les quantificateurs (beaucoup de), les approximateurs (à peu près), les mots « passe-partout » (truc), les marqueurs vagues de catégories (et tout), etc. ainsi que sur leurs fonctions dans différents genres de discours. Le vague est défini comme « highly context-dependent language that lacks specifity » (Ruzaité, 2007 : 53). Même si la pragmatique conçoit un sujet énonciateur en grand partie comme maître de son dire, usant de stratégies communicationnelles pour atteindre un certain but illocutoire, le vague reste néanmoins d'après la plupart des études une propriété de la langue qui se manifeste à travers différentes catégories observables, que celles-ci soient utilisées à dessein ou inconsciemment.

Si ces trois perspectives divergent sur de nombreux aspects, elles convergent sur un point commun :

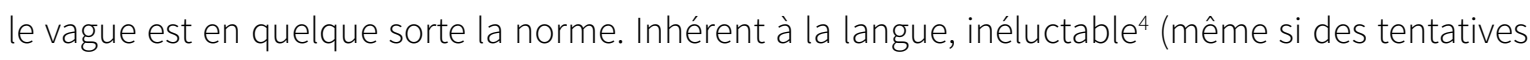
ont été proposées pour développer un système de symboles précis qui remplacerait le langage naturel afin d'éradiquer toute ambiguïté $\left.{ }^{5}\right)$, il constitue « la norme » dans son sens de «normal », voire nécessaire, utile et bénéfique ; il correspondrait alors à une sorte de «norme objective » (Gadet, 2007), scientifiquement mesurable et analysable. L'affaire se présente en revanche tout autrement si l’on « interroge » le locuteur ordinaire.

\section{Le point de vue du locuteur ordinaire : vague comme terme évaluatif}

Afin de pouvoir se pencher sur ce que le locuteur ordinaire considère comme vague dans le discours quotidien et en déceler son positionnement, nous avons examiné l'usage du mot vague dans des séquences telles que "xy est un terme vague, une réponse vague ». Nous avons recherché dans un vaste corpus de presse francophone les termes vague, flou et approximatif ${ }^{6}$ aussi bien employés seuls que pris dans des syntagmes tels que «mot vague », « expression vague », « réponse vague », « concept vague ${ }^{7}$ ». Voici deux exemples:

1. Les recherches en psychologie, au Royaume-Uni, ont montré que la plupart des individus ne se sentent pas personnellement menacés par le changement climatique, parce que c'est pour eux un concept vague, abstrait et difficile à visualiser. (Courrier International, 19 novembre 2009)

2. Autre réponse floue, celle apportée au problème du chômage. La décroissance entraînant une baisse de la production et donc du travail, que faire? « Le plein emploi est une utopie, poursuit Grinevald, il faut donc continuer à réfléchir ». (Libération, 27 septembre 2003)

Dans les deux extraits, les mots vague et flou ainsi que les séquences qui sont jugées vagues (changement climatique ; Le plein emploi est une utopie [...] il faut donc continuer à réfléchir) apparaissent. On pourrait considérer l'attribution du prédicat vague à ces deux séquences comme une simple activité de prédication métalinguistique («X» est vague, tel que « courir» est un verbe). Or, ce jugement, qui est « fondé au moins en

4. "Vague predicates seem to be an unavoidable presence in our language" (Keefe, 2006, 299).

5. Voir Frege, Begriffsschrift, 1882.

6. Les termes vague, flou et approximatif figurent en tant que synonymes dans de grands dictionnaires d'usage : Le Robert, Le TLF, Le Dictionnaire des synonymes, mots de sens voisin et contraires. Afin d'élargir le corpus, nous nous sommes focalisée sur ces trois mots.

7. Afin de pouvoir relever, en plus des termes évaluatifs, les séquences que les locuteurs visaient comme vagues, nous avons cherché majoritairement des syntagmes comportant comme nom tête une expression du « dire » : réponse vague, expression vague, $\boldsymbol{m o t}$ vague, etc. 
partie sur des normes correspondant à une ou des valeurs » (Nadeau, 1999 : 350), permet non seulement de relever les expressions et séquences qui font l'objet du jugement, mais aussi de noter que le terme, dans son usage métalinguistique, revêt une modalité évaluative, car considéré comme jugement appréciatif porté sur la langue (Brunner, 2014).

Yaguello (1988) identifie trois attitudes du locuteur ordinaire vis-à-vis de la langue : explicative, appréciative et normative. Que l'attribution du prédicat vague à une séquence langagière ne relève pas uniquement d'une attitude descriptive ou explicative, mais d'un jugement appréciatif à axiologie péjorative, peut être montré à travers une étude du cotexte immédiat dans lequel apparaît le mot :

3. «Prodigieusement agacé par les réponses floues » du cabinet d’Élisabeth Guigou sur le financement des 35 heures, le sénateur Charles Descours s'en est allé, en effet, faire une enquête sur pièces et sur place. (Le Monde, 2001)

4. Au PS, à La Rochelle, d'autres ont critiqué le trop flou de ses discours [de Ségolène Royal]. (Le Monde, 2006)

5. Un discours flou qui déçoit le monde enseignant. (Libération, 2006)

6. Le flou sarkoziste pour endormir les français. (L'Indépendant, 2008)

7. Si on pouvait, une fois de temps en temps, laisser la caricature, l'outrance et l'approximation de côté. (La République du Centre, 2013)

À travers des verbes illocutoires exprimant l'agacement, la critique, le reproche ou la déception, on peut constater ici à quel point le locuteur attribue une valeur négative au vague, au flou et à l'approximation dans le discours ${ }^{8}$.

Dans un certain nombre d'exemples, on a pu relever les lexèmes situés dans l'entourage immédiat de l'adjectif vague sous forme de coordination (et, mais). L'association avec la valeur négative du mot auquel vague est coordonné à l'aide de la conjonction et, permet au locuteur d'exprimer une évaluation négative. Notre analyse montre alors que la grande majorité de ces constructions de coordination présente le terme vague accompagné d'un adjectif ou d'une proposition à valeur péjorative, alors que les constructions « vague mais x » se caractérisent par une mise en relation entre le terme vague avec un adjectif à valeur positive qui lui est ainsi opposés:

\begin{tabular}{|c|c|}
\hline vague $\underline{\underline{E T}}$ & vague $\underline{\text { MAIS }}$ \\
inopérant & conciliant \\
simpliste & passionnant \\
superficiel & intéressant \\
insuffisant & prometteur \\
\hline
\end{tabular}

8. Deux types de configurations énonciatives se présentent ici : l'une, lorsque le journaliste rapporte les propos d'autres personnes et effectue une interprétation de leurs actes de parole en les qualifiant de reproche ou de critique (exemple 4) ; l'autre, où l'« agacement » est désigné tel quel par l'énonciateur dont les propos sont rapportés (exemples 3). 
L'emploi récurrent du mot vague dans ces cotextes à valeur négative semblent avoir contribué au fait que le terme s'est lui-même chargé de cette modalité appréciative péjorative et que le vague n'a pas bonne réputation auprès du locuteur ordinaire.

Les journalistes à la source des jugements représentent une catégorie de locuteurs ordinaires plutôt aguerrie en matière de langue (style, orthographe, cohérence de texte, etc.) ${ }^{9}$. Mais la fréquence élevée de discours rapportés dans notre corpus dans lesquels le journaliste rapporte les propos de locuteurs d'autres catégories socio-professionnelles - critiquant eux-aussi le vague d'un propos - permet de considérer le corpus comme suffisamment représentatif pour donner une première vision globale de la manière dont le vague est appréhendé par la communauté linguistique francophone.

La haute fréquence de jugements critiques envers le vague positionne le phénomène du côté du horsnormes, ici considéré comme du «mauvais usage »- la précision a contrario comme du «bon usage » correspondant à la norme qu'il convient de suivre. Norme prend ici le sens courant de «normatif » ou " prescriptif » tel que Françoise Gadet (2007) conçoit le concept de « norme subjective ». Le rapport à une même réalité linguistique est donc diamétralement opposé entre locuteurs savants d'une part et locuteurs profanes de l'autre, constat qui n'est en soi pas étonnant étant donné l'approche descriptive dont se réclame généralement le linguiste et qui est beaucoup moins répandue en dehors de ce cercle restreint. L'écart dans l'appréhension du vague incite toutefois à interroger les raisons de ce reproche récurrent à l'encontre du vague dans le discours quotidien.

\section{Les raisons du jugement}

Comment expliquer un tel acharnement contre le vague? Avant de pouvoir avancer une réponse à cette question, une autre convient d'être posée: qu'est-ce qui, dans la séquence jugée vague, amène précisément les locuteurs à émettre leurs jugements ? Concrètement: pourquoi le syntagme "changement climatique » (exemple 1) est jugé vague, pourquoi provoque-il ledit jugement? Afin de pouvoir proposer une réponse, nous allons à présent focaliser sur les différents sens que revêt le terme vague lorsqu'il est employé par le locuteur ordinaire. L'analyse montre (infra) le sens du terme varie en fonction du contexte. L'acharnement contre le vague pourra alors s'expliquer à travers la cristallisation d'un trait commun à tous les sens du mot vague, dénominateur commun qui serait responsable de la critique à l'encontre de ce phénomène. Les exemples suivants permettent de comprendre l'usage hétérogène du terme et ses sens multiples qui en découlent:

1. [...] l'introduction d'un terme pervers, celui de « globalisation » [...], « englobant » en un terme vague et réducteur, sans signification réelle, du moins précise, l'économique, le politique, le social, le culturel [...]. (Le Monde, 22 février 2000)

2. «Multimédia » est désormais un mot un peu cave. Il sert d’euphémisme pour désigner les jeux vidéo, rayons de magasins, noms de filiales ou de filières de formation. C'est surtout un mot vague, utilisé pour nommer quelque chose qu'on ne veut pas limiter à un mot précis : « Je bosse dans le multimédia » est une phrase qui fait mieux que : « Je mets des CD dans des boîtes chez Ubi Soft ». (Le Monde, 10 mars 1999)

9. Ils occupent par conséquent une position plutôt intermédiaire sur un continuum (Paveau, 2008) qui se situerait entre, d'un côté, le locuteur qui produit du savoir populaire à propos de la langue et, de l'autre côté, le locuteur savant (le linguiste) qui produit du savoir scientifique (sans que l'un soit a priori jugé supérieur à l'autre). 
3. Pour autant, le professeur de français se refuse à parler de «crise de l'autorité » à l'école. Il juge l'expression « approximative », fourre-tout. (La Croix, 24 avril 2006)

L'étude du cotexte montre la présence récurrente de mots comme "englober », « désigner », «fourretout» indiquant que les séquences jugées vagues par le locuteur le sont apparemment pour leur caractère «multi-référentiel »; elles n’ont pas de délimitation claire dans leur extension, l'applicabilité référentielle est en cause. Ce sont des expressions qui, comme le dit le locuteur en (8), « englobe » l'économique, le politique, le social, le culturel, renvoient à plusieurs phénomènes ou objet dans le monde et qui, de ce fait, sont jugées vagues. Cette caractéristique multi-référentielle semble être une propriété qui provoque fréquemment ledit jugement. II s'agit là d'un des sens du mot vague - sens qui concorde parfaitement à une des définitions savantes donnée du phénomène (Blay, 2003, supra). Or, ce n'est pas le seul sens; voici d'autres cas de figure :

Marins grecs, officiers français, policiers chypriotes, [...] ont les yeux rivés vers le large. Avec une seule et même question: Quand les bateaux vont-ils arriver de Beyrouth ? Et toujours cette réponse vague : durant la nuit, mais peut-être aussi demain. (24 Heures, 20 juillet 2006).

Quelle est l'ampleur des régularisations que propose, en l'occurrence, la socialiste ? Réponse floue de Sandrine Mazetier, secrétaire nationale chargée de l'immigration : serait concerné "l'essentiel des personnes qui sont en grève en ce moment ". (Libération, 24 novembre 2009).

"Pour le mois de mai, le prix du lait n'est pas arrêté, mais il sera vraisemblablement aux alentours de 220-230 € [...] ». Non satisfaits de cette réponse approximative et du montant estimé, les producteurs annoncent... (Ouest-France, 6 juin 2009).

Autre réponse floue, celle apportée au problème du chômage. La décroissance entraînant une baisse de la production et donc du travail, que faire ? " Le plein emploi est une utopie, poursuit Grinevald, il faut donc continuer à réfléchir. " (Libération, 27 septembre 2003).

Dans ces configurations dialogales (question-réponse), la réponse donnée à la question ne semble pas satisfaisante. Une question pouvant fonctionnellement être décrite comme une requête d'information (Kerbrat-Orecchioni, 2001), on peut alors supposer que lorsque la réponse est qualifiée de «vague », ce jugement signale l'insatisfaction vis-à-vis du contenu informationnel véhiculé par la réponse ${ }^{10}$. II s'agit là d'un usage du mot vague qui apparaît souvent lorsque des expressions telles que « peut-être » (11), « l'essentiel de » (12) ou «vraisemblablement » (13) sont contenues dans les réponses données, expressions qui font partie des catégories de modalisateurs et de quantifieurs également considérées comme catégories du vague dans la littérature (Channel, 1994). Nos données montrent que la grande majorité des jugements contenant le terme vague porte sur la problématique du « manque d'information », ce qui nous conduit à postuler la prise en compte de ce sens lorsque le mot est utilisé dans le discours quotidien.

10. C. Kerbrat-Orecchioni (2001: 95) remarque que l'échange question-réponse « peut être suivi d'une troisième intervention, dite "évaluative" »; c'est ce que l'on trouve lorsqu'une réponse est évaluée comme étant «vague ». 
Les expressions multi-référentielles d'une part, le manque d'informations d'autre part, mais aussi les expressions jugées vagues pour leur trop haut degré d'abstraction, leur relativité contextuelle ou parce qu'elles signalent un déficit épistémique de la part de celui qui emploie le terme en question (dans les constructions avec vague antéposé notamment : "vague idée », « vague connaissance ») constituent autant d'aspects visés par le jugement du vague et forment ensemble les multiples facettes de sens du mot $v_{a g u e}{ }^{11}$. Le trait commun de ces différents sens semblerait alors être celui du manque, de l'absence : les expressions multi-référentielles manquent de frontières précises dans leur extension, les expressions considérées comme abstraites le sont souvent parce que le locuteur déplore le manque d'exemples concrets, la relativité contextuelle implique un manque de point de référence et le manque d'information présente ce trait de manière évidente. Les séquences perçues comme vagues sont celles qui gênent puisqu'elles font toutes preuve de ce manque, propriété connotée péjorativement, qui paraît constituer une entrave à la bonne compréhension.

On pourrait donc avancer que le postulat du bon déroulement de l'échange expliquerait en partie la norme d'un « parler précis » alors que le vague constitue en quelque sorte une entrave au principe de coopération de Grice (1979). Le vague induit, selon une idée partagée, l'incompréhension comme le montre l'extrait suivant:

[...] l'annonce était écrite dans un langage assez vague et bureaucratique pour être carrément incompréhensible. (La Presse Canadienne, 8 décembre 2010).

L'obstacle que présente le vague du point de vue du récepteur lors du processus de décodage, paraît donc conditionner les multiples jugements critiques dans le discours quotidien.

\section{La norme et le mythe de la clarté de la langue française}

Lorsque l'on travaille sur un corpus francophone qui met au jour les divers jugements portés à l'encontre du vague au quotidien, il convient d'avancer une deuxième hypothèse pour expliquer ce positionnement critique des locuteurs : le mythe de la clarté de la langue française, né au xviie siècle (« [...] la clarté du langage, que la Langue Françoise affecte sur toutes les langues du monde » (Vaugelas), « Le génie de cette langue est la clarté et l'ordre » (Voltaire, voir dans Swiggers, 1987). À travers les siècles, l'histoire du français a mobilisé de multiples positionnements normatifs, voire puristes, au sujet de la langue. On pourrait donc considérer que « l'attitude à l'égard de la langue a été si normative qu'elle a contribué à faire du français la langue la plus normée, dit-on, des langues vivantes du monde » (Perret, 2008 : 60). Les multiples remarques à l'égard d'un phénomène linguistique peuvent alors constituer un indice du fait que ce même phénomène se situe hors-normes, et de nos jours encore, l'imaginaire d'une langue claire et précise se trouve actualisé :

Je tiens la justesse et la clarté pour les qualités premières de notre langue [...] à ce point que je doute s'il y a jamais eu, depuis les Grecs, langue plus transparente à la pensée [...]. Or, voilà justement où la langue française a acquis depuis longtemps la réputation d'être incomparable. Bien maniée, elle éclaircit les choses les plus difficiles, et c'est une des raisons de sa longue domination en Europe [...] elle porte plus loin que toute autre l'exigence et la capacité de la clarté. (Duron, 1963 dans Swiggers, 1987 : 15, nous soulignons).

11. Pour une plus ample discussion des différents sens du mot illustrés auprès de divers exemples, voir Brunner (2014). 
Le positionnement des locuteurs contemporains doit alors être situé devant l'arrière-plan des diverses appréciations engagées dans les discours antérieurs politiques et sociaux : « en effet, si tout locuteur peut faire part de sa perception des pratiques langagières, celle-ci est toujours déterminée par du déjà-dit »(Canut, 2007 : 52). En prolongeant cette idée, on pourrait dire que considérer le vague comme mauvais usage suppose un système de valeur préexistant qui se serait établi à la suite des discours autrefois tenus sur la clarté de la langue française.

Les propos du linguiste Harald Weinrich, qui est d'avis que de nos jours la clarté est restée l'attribut favori pour la langue française ${ }^{12}$, confirme cette hypothèse. Le concept de la clarté aurait muté à travers les sciences : en partant d'un concept rhétorique (une virtus dicendi), né dans l'antiquité et impliquant un usage, un style clair de la langue, il se serait transformé au XVII siècle en mythe de la clarté. Le français serait alors la seule langue qui possède cette propriété de manière inhérente. Les locuteurs français auraient, à cause de ce mythe, intériorisé un éthos de clarté (dans le sens d'engagement moral) dans leur manière de s'exprimer, éthos qui s'apparente à un devoir, un impératif plus ou moins inconscient.

L'éthos de clarté va au-delà de l'impératif stylistique tel qu'il est inculqué à toute personne qui écrit ou parle dans les autres pays. L'éthos de clarté a plus de poids, a un pouvoir plus suggestif et pathétique que le simple impératif. Cela peut être imputé au mythe et explique en grande partie pourquoi les Français ressentent et respectent en effet davantage que d'autres nations le devoir à la clarté ${ }^{13}$. (Weinrich, $1961: 541$, notre traduction)

L'affirmation que les Français respecteraient davantage le devoir de clarté que d'autres nations méritent encore d'être prouvée. Que le discours évaluatif à l'encontre du vague peut être ramené à ce " pouvoir suggestif » que constituerait le devoir de clarté, nous semble en revanche être une hypothèse tout à fait légitime. «Des discours évaluatifs naissent souvent de contextes précis liés à l'histoire des individus et des peuples (religion, politique, relations sociales, économiques) mais se transmettent aussi d'imaginaire en imaginaire sans que l'on n'en connaisse plus toujours les raisons. » (Canut, 2007 : 54) - affirmation qui se reflète pleinement dans notre étude d'un phénomène qui se situe hors-normes.

Le hors-normes se trouve dans cette étude exemplifié dans des commentaires métalinguistiques, traces d'un imaginaire de la langue qui se doit d'être précis du point de vue du locuteur ordinaire. Que ce soit pour des raisons qui impliquent le bon déroulement du processus d'encodage et de décodage auquel le vague semble constituer une entrave ou à cause d'un discours inconsciemment intériorisé sur la clarté de la langue française, le discours vague est déprécié au quotidien. Les «normes communicatives » d'une communauté ne transparaissent alors pas uniquement à travers la matérialité discursive produite (Kerbrat-Orecchioni, 2005), mais aussi à travers l'évaluation et la sanction d'un style communicatif qui transgresserait la norme acceptée. Si «l'impératif » de la clarté n'est pas énoncé explicitement, on peut tout de même le déduire des jugements qui sont portés sur son contraire - le discours vague.

12. „Bis auf den heutigen Tag ist die clarté das beliebteste Attribut der französischen Sprache geblieben“" (Weinrich, 1961: 529, notre traduction).

13. „Das Ethos der Klarheit ist mehr als das stilistische Gebot, das den Sprechenden und Schreibenden auch in anderen Ländern nahegelegt wird. Das Ethos der Klarheit hat mehr Gewicht, ist suggestiver und pathetischer als das bloße Gebot. Das dürfen wir dem Mythos zuschreiben, und das erklärt zum großen Teil, warum die Franzosen tatsächlich mehr als andere Nationen die Pflicht zur Klarheit empfinden und erfüllen“"(Weinrich, 1961: 541). 
Pour élargir cette étude et mettre à l'épreuve l'hypothèse du mythe de la clarté de la langue française comme discours sous-jacent aux jugements d'aujourd'hui, il serait en revanche intéressant de se poser la question de savoir ce qu'il en est dans les autres langues : le vague connaît-il le même rejet dans d'autres communautés linguistiques moins marquées par une histoire normative vis-à-vis de la langue que la communauté francophone?

Et que se passe-t-il si l'on observe les jugements portés à l'encontre du vague davantage ciblés dans divers types de discours particuliers (discours politique, juridique ou journalistique) comme l'a fait Laura Calabrese (2016) en étudiant les commentaires des lecteurs sur le discours journalistique dans la presse en ligne. Ces lecteurs « ont tendance à se représenter le discours d'information comme un discours vague et/ ou imprécis », représentations qui seraient « plus ou moins répandues dans la société » (Calabrese, 2016 : 186). Retrouve-t-on ces considérations vis-à-vis d'autres genres discursifs ou va-t-il falloir les nuancer? Quelques pistes donc, qui pourraient contribuer à nourrir la réflexion du hors-normes en général et plus particulièrement par rapport aux discours considérés comme vagues. 


\section{Bibliographie}

Blay Michel (2003), Grand dictionnaire de la philosophie, Paris, Larousse.

Brunner Pascale (2014), Le Vague, die Vagheit. Du mot au concept, pragmatique et folk linguistique, Limoges, Lambert-Lucas.

Brunner Pascale, Husson Anne-Charlotte et Neusius Vera (dir.) (à paraître), Les Carnets du Cediscor, 14, « Les métadiscours des non-linguistes », Paris : Presses Sorbonne Nouvelle.

Calabrese Laura (2016), "Réflechissez avant d'écrire!" Approximation et précision dans le discours des lecteurs de la presse en ligne », H. Bat-Zeev Shyldkrot, S. Adler et M. Asnes, Nouveaux regards sur l'approximation, Paris-Champion, 186-198.

Canut Cécile (2007), «L'épilinguistique en question », G. Siouffi et A. Steuckardt (dir.), Les Linguistes et la norme. Aspects normatifs du discours linguistiques, Bern : Peter Lang, 4972.

Channell Joanna (1994), Vague language, Oxford, Oxford University Press.

Culioli Antoine (1991), Pour une linguistique de l'énonciation. Opérations et représentations, Paris, Ophrys, 9-46.

Dönninghaus Sabine (2005), Die Vagheit der Sprache. Begriffsgeschichte und Funktionsbeschreibung anhand der tschechischen Wissenschaftssprache, Wiesbaden, Harrassowitz Verlag.

Fuchs Catherine (1986), « Le vague et l'ambigu : deux frères ennemis », Quaderni di Semantica, n², 235-245.

Gadet Francoise (2007), La Variation sociale en français, Paris, Ophrys.

Grice Herbert Paul (1979 [1975]), « Logique et conversation », Communications, 30-31, 5772.

Jucker Andreas H., Smith Sara, Lüdge Tanja (2003), "Interactive Aspects of Vagueness in Conversation", Journal of Pragmatics, 35, 1737-1769.

Keefe Rosanna (2000), Theories of Vagueness, Cambridge, Cambridge University Press.

Keefe Rosanna (2006), "Vagueness: Philosophical Aspects", Encyclopedia of Language and Linguistics, 13, Amsterdam, Elsevier, 298-301.

Kerbrat-Orecchioni Catherine (2001), Les Actes de langage dans le discours, Paris, Nathan.

Kerbrat-Orecchioni Catherine (2005), Le Discours en interaction, Paris, Armand Colin.

Kleiber Georges (1987), "Quelques réflexions sur le vague dans les langues naturelles », S. Mellet (dir.), Études de linguistique générale latine: offertes en hommage à Guy Serbat. Paris, Société pour l'information grammaticale, 57-172.

Lecolle Michelle (dir.) (2014), Métalangage et expression du sentiment linguistique «profane», Le discours et la langue, $n^{\circ} 6-1$.

Mihatsch Wiltrud (2010), "Wird man von hustensaft wie so ne art bekifft ?", Approximationsmarker in romanischen Sprachen, Frankfurt am Main, Vittorio Klostermann.

P. von Münchow et F. Rakotonoelina, (2006), « Discours, cultures, comparaisons », Les Carnets du Cediscor, $n^{\circ} 9$. 
Niedzielski Nancy A. et Preston Dennis R. (2000), Folk Linguistics, Berlin, Mouton de Gruyter.

Overstreet Maryann (2005), "And stuff und so: Investigating pragmatic expressions in English and German", Journal of Pragmatics, 37, 1845-1864.

Paveau Marie-Anne (2008), « Les non-linguistes font-ils de la linguistique ? Une approche anti-éliminativiste des théories folk», Pratiques, n¹39/140, 39-110.

Perret Michèle (2008), Introduction à l'histoire de la langue française, Paris, A. Colin.

Ruzaité Jūratè (2007), Vague Language in Educational Settings, Frankfurt am Main, Peter Lang.

Swiggers Pierre (1987), «À l’ombre de la clarté française », Langue française, n 75, 5-21.

Weinrich Harald (1961), Die clarté der französischen Sprache und die Klarheit der Franzosen“, Zeitschrift für romanische Philologie, $n^{\circ} 77,528-544$.

Williamson Timothy (1994), Vagueness, Londres /New York, Routledge.

Wilmet Marc (dir.) (1987), « La clarté française », Langue française, n 75.

Yaguello Marina (1988), Catalogue des idées reçues sur la langue, Paris, Seuil. 